\title{
Utilisation des traceurs pour les études d'implantation des émissaires en mer
}

\author{
PAR
}

M. Gauthier

Chef du Service Scientifique de SOGREAH
ET B. Quetin
Ingénieur au Service Scientifique de SOGREAH

\section{Introduction}

Les études de localisation des émissaires en mer conditionnent des investissements importants et sont soumises à des contraintes variées en fonction de la nature des ouvrages, d'une part, de l'usage des eaux littorales, d'autre part. Elles impliquent toujours une prévision de l'impact des rejets pollués sur le milieu récepteur.

Ce deuxième aspect des problèmes nécessite un certain nombre de mesures in situ pour prévoir les zones d'atteinte des pollutions et les concentrations associées à différentes échelles de temps. Compte tenu de la variabilité des phénomènes étudiés, ces mesures seront le plus souvent utilisées pour régler les paramètres des modèles. Ceux-ci permettront de prévoir les situations les plus critiques et de comparer, de ce point de vue, différentes solutions de rejet, ou bien de fixer des objectifs minimaux d'épuration préalable.

Ces mesures sont basées sur le marquage de masse d'eau pour lesquelles nous disposons de différentes techniques: marqueurs radioactifs, biologiques, chimiques ou flotteurs. Notre exposé sera centré sur les deux marqueurs les plus utilisés, c'est-à-dire les flotteurs, d'une part, les colorants, d'autre part, encore d'une grande partic des problèmes abordés se retrouvent dans l'usage d'autres marqueurs.

Avant d'entrer dans la technologie et l'analyse des problèmes spécifiques, il convient d'identifier ce que l'on veut mesurer et les modes d'actions qui interviennent.

Nous nous proposons, pour un rejet continu, de prévoir où iront les pollutions à des échelles de temps de quelques heures jusqu’à la journée et de connaître les concentrations obtenues.

Les zones d'atteintes seront conditionnées par les trajectoires des masses d'eau polluées et leur dispersion, les concentrations par les dilutions et, éventuellement, les effets d'autoépuration du milieu. L'ensemble est donc en dépendance très étroite de l'hydrodynamique dans la zone et peut se subdiviser en quatre aspects :

- la convection, c'est-à-dire le déplacement moyen des masses d'eau (centre de gravité d'une tache colorée, par exemple) :

la diffusion turbulente qui n'agit, près des côtes, qu'à des échelles faibles devant la convection ;

- la déformation locale des masses d'eau qui, associée à la diffusion turbulente, en accroit les effets dans de larges proportions ;

- la convection différentielle : provoquée par les hétérogénéités diverses (topographies, vents). Elles induisent des effets dispersifs qui, dans certains cas, sont encore d'un ordre de grandeur supérieur.

Il est important de souligner le caractère aléatoire des phénomènes dispersifs, d'une part à cause de la turbulence, d'autre part à cause de l'influence des conditions météorologiques et principalement du vent. Nous en avons une image plus habituelle en examinant les panaches de fumées qui, à des échelles différentes, reproduisent des phénomènes physiquement très semblables. Les implications au niveau des mesures conduisent :

- à en accroître le nombre pour pouvoir saisir les paramètres de variabilité ;

- à la nécessité de protocoles de mesures précis et complets ;

- à porter un effort sur l'interprétation des mesures par l'usage de différents modèles. 


\section{Les flotteurs}

Les flotteurs constituent un premier type de marqueur intéressant et économique. Ils sont surtout utiles pour l'étude du transport de masse à grande échelle. Très pratiques pour les mesures en surface et à faible profondeur, ils deviennent toutefois onéreux et difficiles d'emploi pour des mesures audelà de 5 à 10 mètres de profondeur.

De très nombreux modèles ont été utilisés, allant de la carte "siphonophore" (du nom d'une petite méduse) aux flotteurs de Swallow, en passant par les flotteurs à "drogue" (du mot anglais : drag).

Les flotteurs seront d'autant plus utiles que les écoulements sont lents. Il faut les utiliser davantage dans le but de déterminer des trajectoires moyennes que pour des mesures de vitesse sur de courtes périodes de temps. Il est indispensable de multiplier les flotteurs et de concevoir les mesures comme une statistique. A titre indicatif, nous avons, au cours d'expériences en Méditerranée, suivi simultanément jusqu'à 20 flotteurs à raison d'un repérage toutes les demi-heures pendant 5 à 10 heures. L'usage des flotteurs isolés est en effet aléatoire, eu égard aux fluctuations du champ des vitesses évoqué précédemment. De même, l'usage de flotteurs de surface ne peut pas rendre compte du profil de vitesse ou du rôle de la force de Coriolis.

En particulier, les cartes "siphonophores" ou les "flotteurs homothétiques" ne peuvent être représentatifs que des pollutions par les corps flottants de petite dimension et en aucun cas du devenir d'une couche d'eau polluée dont l'épaisseur n'est jamais faible et croît du fait de la diffusion verticale. Il est donc essentiel de mesurer le déplacement en fonction du temps à différentes profondeurs et surtout sur les premiers mètres, en utilisant des flotteurs dont les drogues seront immergées en conséquence ; on est toutefois limité dans cette voie par l'erreur croissante faite sur la mesure, puisque la différence de vitesse entre la surface et le niveau d'immersion de la drogue croît, ou par les risques d'accrochage sur le fond lorsque les profondeurs sont faibles et variables (mer à marée).

La présence d'un profil de vitesse à proximité de la surface de l'eau, et tout particulièrement si celui-ci est dû au vent, rend la conception des flotteurs beaucoup plus difficile qu'il peut paraître a priori. Elle suppose un calcul précis des forces de trainée et de poussée agissant sur l'élément flottant et sur la drogue. L'ensemble se déplace à une vitesse intermédiaire entre celle régnant au niveau du flotteur et celle régnant au niveau de la drogue. Il faut donc dimensionner l'appareil et calculer les traînées pour minimiser la dérive. En ce sens, il est souvent nécessaire de supprimer toute prise au vent, ce qui complique alors la recherche des flotteurs en mer et suppose l'appui d'un avion de reconnaissance ; c'est un inconvénient notable, mais non prohibitif. II n'est pas exclu de transformer les flotteurs soit en émetteur radio, soit en répondeur radar, mais on aura alors les difficultés pour individualiser chacun des appareils.
A contrario, une série de flotteurs, dont les drogues sont immergés à différentes profondeurs rendra compte du profil vertical des vitesses, du rôle de la force de Coriolis et ces éléments seront essentiels pour l'appréciation de la dispersion dans le plan vertical défini par l'écoulement. A ce titre, il est intéressant de coupler ces mesures avec une mesure de diffusion turbulente dont on parlera plus loin.

La pérennité des flotteurs permet les mesures de longue durée. C'est un avantage essentiel pour les mesures à caractère biologique, ou chimique, d'autant que certaines analyses chimiques sont impossibles en présence d'un colorant.

Signalons enfin que certains expérimentateurs ont réalisé des mesures de diffusion turbulente en larguant un très grand nombre de flotteurs qui étaient ensuite soigneusement repérés sur des photographies aériennes. Ces données étaient alors traitées par ordinateur pour reconstituer le centre de gravité de la tache, les ellipses théoriques d'iso-concentration, et les coefficients de diffusion horizontale.

\section{Usage des colorants}

Nous limitons notre exposé à l'usage des colorants bien qu'on puisse arriver à des résultats comparables avec des traceurs radioactifs. L'emploi de ces derniers est en outre largement traité par ailleurs.

Sur des durées de quelques heures, les colorants permettent des mesures précises de dilution et de dispersion et ont l'avantage sur d'autres marqueurs de pouvoir être repérables visuellement. Cet avantage est considérable si l'on tient compte des échelles d'espace relatives de convection et de dispersion.

Le principe consiste à mesurer l'évolution dans le temps d'une tache de colorant de préférence par photographie aérienne. Simple en apparence, cette mesure est, en réalité, d'une grande complexité : des améliorations notables de cette technique ont été réalisées par Sogreah, en particulier dans le cadre d'études méthodologiques effectuées pour le compte du Service Central Hydrologique et du CNEXO.

\section{La Rhodamine B : avantages et inconvénients}

Le colorant de loin le plus utilisé en mer est la Rhodamine B. Il n'y a pas lieu d'insister sur son pouvoir colorant, ni sur les possibilités de dosage jusqu'à des teneurs extrêmement faibles, encore, qu'en pratique, il soit préférable de se limiter à un ou deux ordres de grandeur au-dessus de la limite théorique.

La Rhodamine est un produit sans toxicité connue ni pour les utilisateurs, ni pour le milieu marin, c'est un colorant basique, donc chimiquement assez stable dans l'eau de mer. Son spectre de réflectance présente une forte dominante dans les longueurs d'onde supérieures à 600 nanomètres*,

* 1 nanomètre $=1000$ microns $=10$ angströms

\begin{tabular}{|c|c|c|c|c|c|c|c|c|c|c|}
\hline Longueur d'onde en nanomètres & 340 & 380 & 420 & 460 & 500 & 540 & 580 & 620 & 660 & 700 \\
\hline $\begin{array}{l}\text { Coefficient moyen d'absorption } \\
\text { par metre de profondeur }\end{array}$ & 0,38 & 0,148 & 0,041 & 0,015 & 0,016 & 0,024 & 0,055 & 0,178 & 0,840 & 2,400 \\
\hline Facteur spectral de luminance $(\%)\left({ }^{1}\right)$ & & 24 & 36 & 52 & 40 & 25 & 27 & 55 & 81 & 82 \\
\hline
\end{tabular}

(1) Le facteur spectral de luminance donne le pourcentage d'énergie lumineuse réfléchie par une surface colorée. Il est mesuré par comparaison avec un diffuseur parfait d'oxyde de magnésie dont la réflectance est égale à $100 \%$. 
c'est-ä-dire dans l'orange, le rouge et le proche infrarouge ; ces couleurs contrastent bien avec la couleur de la mer, d'où une excellente visibilité et des conditions intéressantes en cas de prise de vues aériennes. A contrario, l'eau de mer a un coefficient d'absorption de la lumière qui croît au contraire pour ces mêmes longueurs d'onde et il faut s'attendre a une forte réduction de la visibilité de la Rhodamine dès que celle-ci se situe à quelques mètres de profondeur.

La Rhodamine a aussi quelques inconvénients :

- parce qu'elle est utilisée dans l'industrie, notamment en teinturerie et en papeterie, et qu'on en retrouvera dans certaines eaux littorales;

- parce qu'elle est particulièrement salissante en cours d'emploi et que la préparation des solutions suppose de grandes précautions ;

parce qu'elle est conditionnée soit en poudre, ce qui exige sa dilution préalable, soit en solution dans l'acide acétique, mais la densité de cette solution est plus grande que celle de l'eau de mer, ce qui nécessite également une préparation ;

... enfin, si les eaux sont fortement chargées en matières colloïdales, on peut redouter des phénomènes d'adsorption qui ne sont pas toujours très faciles à apprécier.

\section{Conditions de déversement de la rhodamine}

Une connaissance précise des conditions initiales de déversement de la Rhodamine est fondamentale, car le champ des concentrations ultérieures en dépend très étroitement. Par ailleurs, l'équation de la diffusion n'est pas d'un maniement très simple et, hormis quelques modes d'injection simples, on aboutira à des calculs inextricables pour exploiter les résultats.

Deux solutions sont possibles, le déversement en continu à débit constant ou l'injection unique sous forme de tache. La première solution peut, a priori, paraitre séduisante dans la mesure où elle simule bien un rejet réel. Sans la condamner totalement, nous pensons qu'elle présente de nombreux inconvénients. Si l'on veut en effet suivre suffisamment longtemps le panache résultant, le débit de colorant nécessaire sera notable. Il est, par ailleurs, illusoire de vouloir atteindre un régime permanent à moins de maintenir ce débit pendant plusieurs jours. Il est facile de s'en rendre compte à partir de la solution correspondante de l'équation de la diffusion écrite en variables Lagrangiennes (axes liés au centre de la tache) :

$$
c=\frac{Q}{2 \pi K r}\{1-\operatorname{erf}(r / \sqrt{4 K t})\}
$$

dans laquelle :

$$
\begin{aligned}
r= & \text { distance au centre } \\
t= & \text { temps } \\
Q= & \text { débit matière de colorant } \\
c= & \text { concentration en un point quelconque de coordonnées } \\
& x, y, z, t \\
K= & \text { coefficient de diffusion (diffusion ici supposée isotrope, } \\
& \text { pour simplifier l'écriture). }
\end{aligned}
$$

Le régime permanent sera obtenu quand le terme $\operatorname{erf}(r / \sqrt{4 K t})$ devient négligeable ; soit, compte tenu du développement en série de la fonction d'erreur au voisinage de zéro :

$$
\operatorname{erf} .(u) \cong \frac{2 \cdot u}{\sqrt{\pi}}
$$

quand précisément $r / \sqrt{4 K t}$ devient négligeable. Le temps $t$ doit alors être de l'ordre du carré de la distance à la source. Cette méthode est donc très onéreuse ; aussi vaut-il mieux dissocier la matérialisation de la dérive d'un panache et la mesure de la diffusion turbulente.

La méthode des "taches" est bien préférable. Elle consiste à injecter de manière aussi ponctuelle et instantanée que possible, une certaine quantité de colorant en solution d'une densité égale à celle de l'eau de mer, le plus souvent comprise entre 1 et $10 \mathrm{~kg}$ de Rhodamine B. L'idée directrice de la méthode repose sur la solution la plus simple de l'équation de la diffusion dite de la "source ponctuelle instantanée" :

$$
c=\frac{2 M}{\sqrt{4 \pi t)^{3} K x \cdot \bar{K} y \cdot K z}} \exp .\left\{-\frac{1}{4 t}\left(\frac{x^{2}}{K_{x}}+\frac{y^{2}}{K_{y}}+\frac{z^{2}}{K_{z}}\right)\right\}
$$

dans laquelle :

$$
\begin{aligned}
x, y, z \quad & \text { coordonnées spatiales Lagrangiennes, l'axe } x \\
& \text { orienté dans le sens de l'écoulement; } \\
K_{x}, K_{y}, K_{z}= & \text { coefficients de diffusivité dans les directions } \\
& \text { des axes correspondants; } \\
= & \text { temps; } \\
t \quad & \text { Masse de colorant injectée ; } \\
M \quad & \text { concentration en un point quelconque } x, y, t .
\end{aligned}
$$

Mais on doit s'attacher à reproduire par le mode opératoire, du moins à la précision utile, les conditions initiales conduisant à cette formulation, soit :

$$
\begin{gathered}
c=0 \forall t \leqslant 0 \\
c=\delta \quad \grave{a} \quad x \quad y=z=t=0 \quad \text { (fonction de Dirac) }
\end{gathered}
$$

\section{Technologie des mesures}

La technologie des mesures n'est pas figée et suppose encore des progrès. Néanmoins, elle ne s'improvise pas et c'est ce que nous voulons montrer en évoquant quelques aspects importants.

Il faudrait tout d'abord s'affranchir de tout bateau ou du moins des bateaux ayant une trainée notable. En effet, l'énergie transmise par les hélices est entièrement dissipée en turbulence supérieure de plusieurs ordres à celle que l'on veut mesurer. Il faut donc réaliser l'injection du colorant à distance et utiliser soit des mesures à distance soit la photographie aérienne sous réserve d'en faire un procédé de mesure quantitatif.

Remarquons tout de suite que l'on peut se contenter de coordonnées Lagrangiennes ce qui ne nécessite pas le rattachement des photographies à des bases géographiques terrestres, seule la mise à l'échelle du cliché et la verticalité de l'axe de prise de vue importent. La technique nécessaire est donc un peu plus simple que la photogrammétrie classique.

L'analyse photodensimétrique des clichés est hautement souhaitable mais elle suppose des prélèvements pour dosage et malheureusement l'intervention d'un bateau, à moins d'imaginer un appareillage de prélèvements à distance. Quelle que soit la méthode, le prélèvement doit être synchronisé avec une prise de vue aérienne ; on assure ainsi un bon repérage en plan du point de prélèvement. 
La diffusion verticale ne peut pas se déduire des photographies et des prélèvements directs sont nécessaires. Il est hautement souhaitable pour l'interprétation ultérieure que ces prélìvements soient effectués à différentes profondeurs mais simultanément (et avec repérage en plan sur une photographie aérienne). On peut toutefois les différer jusqu'à la fin de l'expérimentation car la diffusion verticale est toujours beaucoup plus faible que la diffusion horizontale ; ceci permet d'intervenir dans la tache colorée lorsque celle-ci est déjà grande et la perturbation introduite par un bateau un peu moins sensible. L'intérêt d'un colorant, bien visible dans son ensemble, est alors évident car il montrera à l'expérimentateur averti les risques pris ou les incidents de manipulation.

Nous avons insisté précédemment sur les conditions initiales de la mesure. Il faut aussi insister sur les paramètres conditionnant l'hydrodynamique sur la zone considérée. Il est indispensable de connaitre parallèlement au développement de la tache :

- la marée,

le vent,

- La répartition des vitesses en fonction de la profondeur, - les stratifications éventuelles d'origine saline ou thermique.

Ces deux dernières notions sont d'importance capitale. En effet, la diffusion turbulente verticale est directement liée au gradient vertical des vitesses en raison même de sa définition. Par ailleurs, l'existence d'une stratification saline ou thermique est de nature à inhiber complètement la diffusion verticale. Une stratification constitue une frontière du domaine dans lequel s'effectue la diffusion ; la validité de l'équation 2 relative à un domaine semi-infini peut aiors être éventuellement remise en cause.

\section{Interprétation des mesures}

L'interprétation des photographies aériemnes n'est pas tout à fait un problème plan, car, du fait de la pénétration de la lumière dans l'eau de mer, les clichés obtenus ne donnent pas unc image de la concentration en surface, mais le résultat d'une sommation pondérée le long de chaque verticale. On peut se proposer d'évaluer cet effet et le terme correcteur à introduire dans l'équation 3.

Si on appelle :

$\lambda \quad$ la longueur d'onde de la lumière

$\chi(\lambda)$ le coefficient moyen d'absorption de l'eau de mer

$\beta(\lambda)$ le facteur spectral de luminance de la Rhodamine B,

l'image photographique d'une particule liquide colorée située à la profondeur $z$ est atténuée dans le rapport:

$$
f(z)=\int_{400}^{700} \beta(\lambda) \exp [-2 \chi(\lambda) z] d \lambda / \int_{400}^{700} \beta(\lambda) d \lambda(4)
$$

et en définitive la concentration apparente sur les clichés est ćgale à :

$$
\begin{array}{r}
c^{\prime}=\frac{2 M}{\sqrt{(4 \pi t)^{3} K x \cdot K y \cdot K z}} \exp \left[-\frac{1}{4 t}\left(\frac{x^{2}}{K x}+\frac{y^{2}}{K y}\right)\right] \\
\int_{0}^{\infty} f(z) \exp \left(-\frac{z^{2}}{4 K z t}\right) d z(5)
\end{array}
$$

Il n'y a pas de solution analytique exacte à ce probleme car :

les fonctions $\chi(\lambda)$ et $\rho(\lambda)$ sont expérimentales et non formulées analytiquement,
- la fonction $\chi(\lambda)$ dépend de l'état de la meret des matières en suspension qu'elle contient,

même si l'on tente un ajustement de $f(z)$ sur une fonction analytique, celle-ci doit être telle que la seconde intégrale en $z$ soit possible.

On peut donc proposer soit une tabulation numérique, soit un ajustement approché de la forme :

$$
f(z)=-0,175 \sqrt{z}+0,851
$$

On obtient alors pour le calcul de la diffusion turbulente horizontale $K y$ :

$$
K y=\frac{y_{i+1}^{2} / t_{i+1}-y_{i}^{2} / t_{i}}{4\left[\log \left(t_{i} / t_{i+1}\right)+\log \left(A_{i+1} / A_{i}\right)\right]}
$$

dans laquelle la quantité $A i$ sera, soit lue dans la table ci-après obtenue par intégration numérique directe, soit calculée par la formule approchćc déduite de 6.

$$
\begin{gathered}
A(4 K z t)=\int_{0}^{\infty}(0,851-0,175 \sqrt{z}) \exp \left(-z^{2} / 4 K z t\right) d z \\
A_{i}=0,851-0,121\left(4 K z t_{i}\right)^{1 / 4}
\end{gathered}
$$

\begin{tabular}{|l|c|c|c|}
\hline $4 K z t$ & $A(4 K z t)$ & $4 K z t$ & $A(4 K z t)$ \\
\hline 0,20 & 0,3329 & 12 & 1,8320 \\
0,40 & 0,4512 & 14 & 1,9431 \\
0,60 & 0,5375 & 16 & 2,0439 \\
0,80 & 0,6079 & 18 & 2,1364 \\
1 & 0,6684 & 20 & 2,2222 \\
2 & 0,8938 & 25 & 2,4131 \\
4 & 1,1875 & 30 & 2,5788 \\
6 & 1,3973 & 35 & 2,7260 \\
8 & 1,5653 & 40 & 2,8588 \\
10 & 1,7076 & 45 & 2,9802 \\
\hline
\end{tabular}

L'incidence de cette correction atteint 8 à $13 \%$, elle croit avec le temps.

L'interprétation de la diffusion turbulente verticale devient particulièrement difficile dans les écoulements à fort gradient vertical de la vitesse, comme cela est le cas dès que le vent devient le moteur principal de cet écoulement. Le calcul de $K z$ suppose alors le recours à des modèles mathématiques particulièrement délicats et qui nécessitent une connaissance suffisante de la répartition verticale des vitesses.

\section{Conclusions}

Les études en matière de pollution supposent l'accès à une connaissance beaucoup plus fine des écoulements que ce qui était jusqu'ici nécessaire en matière de navigation et d'ouvrage à la mer. Curieusement les techniques de mesure font appel à des moyens rustiques, flotteurs et colorants, mais cette rusticité ne doit pas faire oublier la complexité des phénomènes et celle des essais in situ. Nombre et simultanéité des mesures supposent des moyens importants et malheureusement des coûts élevés qui ne compensent qu'en partie Ie prix de revient modeste des flotteurs et des colorants. Cela conduit dans la pratique à porter un effort particulier aux problèmes d'exploitation et d'interprétation de ces mesures. 


\section{Bibliographie}

LACOMBE -- Cours d'océanographie physique, Gauthier Villars 1968. NEUMANN - PIERSON - Principles of physical Oceanography. Pren tice Hall Inc. 1966.
GAUTHIER (M.F.) - Introduction à l'étude des rejets thermiques dans l'océan : dispersion dans les courants La Houille Blanche - Juillet - Août 1974.

QUETIN - Evolution d'une eau polluée rejetée en mer (SHF 13èmes journées de l'Hydraulique; Paris 1974).

Documentation technique de la Société Française des Matières Colorantes.

\section{Discussion}

Président : M. Jean RoDIER

M. Le Président remercie M. QUETIN de son exposé et souligne son aspect tout à fait pratique, témoignage de la solide expérience de ses auteurs et ouvre la discussion.

M. MOLIN A R I présente les observations, ci-après

"Nous pourrions présenter des cas de dispersion par diffusion dans lesquels les discordances entre les directions d'écoulement de la couche pelliculaire et de la tranche sous-jacente sont encore plus marquées que dans les expériences relatées par M. QUETIN. Nous avons, en effet, observé des écarts de direction dépassant $90^{\circ}$ au cours de nombreux essais effectués, en Méditerranée notamment, à des distances du littoral variant entre plusieurs centaines de mètres et plusieurs kilomètres. Ces discordances résultent évidemment de l'effet du vent qui -surtout dans les régions méditerranéennes - subit des sautes brutales en direction et en intensité.

"Ie voudrais maintenant faire une remarque sur la technologie des essais. Il est possible que le bombardement de la surface depuis un avion avec un flacon contenant le traceur ne soit pas la solution idéale il serait, sans doute, préférable d'opérer depuis une embarcation et par exemple, de crever un boudin (contenant le traceur) disposé de façon à intéresser une tranche d'eau verticale suffisamment profonde.

"En effet, dans le cas d'un rejet en mer, compte tenu de la présence éventuelle d'une thermocline, les eaux douces légères, après avoir subi parfois une très faible stratification, n'arrivent en surface qu'après avoir subi une certaine dispersion sur chaque tranche verticale".

\section{QUETIN répond}

Tout d'abord je précise que le colorant n'est pas laché depuis l'avion, mais à partir d'un bateau : il est préparé dans un récipient en verre rempli de telle sorte qu'il flotte.

L'injection du traceur au moyen d'un boudin vertical a été réalisée, je crois, par le C.E.A. à la LA HAGUE, avec le concours de Monsieur HYACINTHE alors à la Faculté de GRENOBLE. Cette solution est possible si les vitesses sont assez homogènes sur toute la hauteur (comme à LA HAGUE) et ramène à un schéma à deux dimensions.

Pour la simulation et l'étude du panache ascensionnel, nous pensons que le marquage est très difficile voire inutile. En effet, à la suite des très nombreux essais sur modèle réduit, on dispose aujourd'hui de formules suffisamment classiques pour calculer les principales caractéristiques de ce panache.

Il ne faut pas perdre de vue qu'un panache ressemble à de la fumée de cigarettes constituée d'une série de bouffées dont les mouvements relatifs sont aléatoires. Les schématisations habituelles par des répartitions gaussiennes ne sont vraies qu'en moyenne sur un certain laps de temps. L'émission d'une bouffée de colorant en profondeur donnera à la surface quelque chose d'imprévisible et l'on ne connaîtra pas la condition initiale pour l'étalement ultérieur. Des expériences récentes faites par émission dans les émissaires d'Hyères et de St-Tropez aboutissent à des taches présentant un minimum de concentration au centre et qui sont absolument ininterprétables.

Notre position serait plutôt de dissocier les problèmes : d'une part le panache ascensionnel que l'on sait étudier, d'autre part la dispersion en surface. Conformément même aux équations, celle-ci se décompose encore en termes de transport et de diffusion. Nous dissocions ces deux parties et nous mesurons la diffusion avec des taches de Rhodamine.
Pour simuler un rejet, dit M. MOLINARI, il faudrait s'intéresser, non seulement à la couche pelliculaire -qui est extrêmement influencée par le vent-mais aussi à la couche sub-corticale influencée par la houle et éventuellement ensuite à la tranche inférieure qui est évidemment beaucoup moins concernée par le rejet d'eas douce. Lors d'un marquage superficiel -par éclatement d'un récipient comme vous l'avez indiqué- je crois qu'il faudrait prendre certaines précautions pour ne pas se limiter à une injection à la surface et procéder à un marquage intéressant une épaisseur d'au moins deux mètres ou trois mètres d'eau

\section{QUETIN précise}

Après une heure et demie, les taches de Rhodamine que vous avez vues sur les photographies s'étendaient jusqu'à 10 mètres de profondeur. La diffusion verticale était calculée à partir d'une série de prélèvements faits tous les 1 à 2 mètres jusqu'à -10 mètres. Mais, du fait du fort gradient de vitesse à proximité de la surface nous avons dù mettre au point un programme de calcul extrêmement complexe pour éviter les problèmes d'ordres numériques propres à ce type de modèle. Nous avons trouvé un bon accord avec les courbes théoriques de diffusivité que j’ai projetées.

En pratique, si l'on travaille en vue d'un rejet futur on n'a aucune idée, a priori, de l'épaisseur de la couche polluée, il faut donc faire des mesures sur une certaine profondeur jugée raisonnable. De fait, un panache n'est pas très épais au début de son étalement.

M. le Professeur LACOMBE pose quelques questions au cours d'une intervention qu'il a résumée comme suit :

1) Comment mesurez-vous les vitesses de courant tout près de la surface?

2) On a évoqué, à juste titre, l'effet de la stratification dûe au fait que l'eau de l'exutoire a une densité toujours inférieure à celle de l'eau de mer. Mais à quelque distance des côtes, les phénomènes de transfert et d'échange de chaleur, de vapeur d'eau et de quantité de mouvement à la surface interviennent-ils pour créer la "mixed layer" qui se trouve au-dessus de la thermocline et dans laquelle le mouvement varie peu en profondeur?

3) Vous avez dit que les modèles théoriques de courants côtiers engendrés par le vent ne prennent pas en compte la force de Coriolis. Dans la réalité, il faut évidemment en tenir compte.

4) Finalement, la méthode de traceurs préconisée sert à determiner des valeurs des coefficients de diffusion turbulente. Ceci, bien entendu, ne dispense pas de faire des mesures de la "convection" c'est-à-dire des courants. Ceux-ci étant très variables, doivent être l'objet de mesures de longue durée en plusieurs points et à plusieurs niveaux ; ces mesures doivent être accompagnées de mesures thermiques (stratification) et de mesures de vent sur la région. Ensuite, il convient de mettre au point des modèles explicatifs destinés à la compréhension des mécanismes de la génération des courants".

M. QUETIN répond en ces termes, aux questions posées par $M$. le Professeur LACOMBE :

Les vitesses de courant en surface étaient mesurées avec des flotteurs à drogue à des profondeurs successives de $0-0,20-0,50-1-2-3$ et 5 mètres. 
Les vues projetées sont relatives à une étude dans une darse portuaire et on a négligé la forme de CoR IOLIs dans les modèles. Mais nous disposons d'un modèle similaire qui en tient compte et qui met en évidence des écoulements en hélice proches de la théorie d'EKMAN.

M. Paul Ch. LeVEque (Université de BordeAuX I) apporte d'intéressantes informations sur les résultats d'analyse de tritium, obtenus sur une "coupe" verticale jusqu'à $4400 \mathrm{~m}$ dans le Golfe de GASCOGNE Les activités en tritium sont très variables et on a observé des chiffres de 0 à 4 U.T. en profondeur, puis deux passages, notamment à $1200 \mathrm{~m}$, à très faible activité (soit le bruit de fond, soit des chiffres de 1 à 2 U.T.), enfin des valeurs dispersées et en surface des activités de 12 à 17 U.T. Par ailleurs, nous avons pu retrouver, grâce aux analyses de tritium, le pana che de la Gironde à 150 ou $200 \mathrm{~km}$ vers l'Ouest.

Je serais heureux d'avoir communication des mesures effectuées par M. Paul Ch. Leveque, dit M. le Professeur LACombe; il existe, en effet, dans cette région, entre 800 et 1200 mètres de profondeur, une couche très marquée par l'eau de la Méditerranée; il serait assez logique d'observer une différence de teneur en tritium vers ces niveaux.

\section{GUIZERIX intervient alors en ces termes :}

"Je ferai un commentaire en trois points sur l'exposé de M. QUENTIN et la discussion qui l'a suivi.

"Je me souviens que vers 1967 l'A.I.E.A. avait commandité une étude qui fut réalisée par l'Isotopen Centralen de COPENHAGUE. Son objet était de tester la validité de 5 à 6 modèles diffusionnels qui étaient jugés, à ce moment là, les meilleurs. L'étude porta sur un patrimoine expérimental riche de 180 à 200 campagnes dont disposait ce laboratoire. La conclusion fut qu'aucun des modèles ne parvenait à décrire les phénomènes observés. Je crois que cela était dû à une insuffisance de connaissance de la convection sur laquelle on vient "greffer" la diffusion. Cette indigence est le premier des maux dont souffrent les modèles diffusion nels en mer, et l'on peut penser que les traceurs ont un rôle décisif à jouer pour rectifier cet état de fait.

"Je soulignerai, en second lieu et après M. QUETIN, l'importance des courants épidermiques, disons des courants compris dans une zone de $20 \mathrm{~cm}$ sous la surface de l'eau. Ces derniers qui dérivent, à court terme du vent, sont évidemment très importants dans les problèmes de pollution. Il ne semble pas qu'ils soient pris en compte dans les modèles, et des études à leur sujet paraissent s'imposer. Nous avons effectué dans l'Atlantique, au large d'ARCACHON, une trentaine de campagnes de traceurs, en collaboration avec le Laboratoire Central d'Hydraulique de France. Nous réalisions pour chacune une double injec tion de traceur, d'une part, de flotteurs en bois, pratiquement insensibles au vent, et d'autre part de rhodamine $B$ à moins $1 \mathrm{~m}$. Dans $50 \%$ des cas, les flotteurs en bois ont pris des directions différentes, parfois opposées à celle de la rhodamine $B$.
"M. QuETIN ne paraît pas, par ailleurs, très favorable à la définition du nuage de traceurs à l'aide d'un appareil porté par un bateau qui effectue une navigation en zig-zag et semble lui préférer la photographie aérienne. Dans nos expériences, tant en Méditerranée que dans l'Atlantique, nous n'avons pas, avec des embarcations de 15 à $20 \mathrm{~m}$, observé de perturbations du nuage de traceur. L'exploration doit évidemment commencer seulement lorsque ce dernier a déjà une dimension assez grande"

"Je suis assez d'accord répond M. QuETin avec la plupart de vos remarques. Il faut tenir compte de la taille de la tâche et de l'importance de la diffusion turbulente sur le site considéré. Ceci soulève implicite ment le débat entre les diffusions dues, soit au cisaillement dans l'écoulement, soit à la turbulence libre qui conduit à la loi des $4 / 3$ laquelle est d'ailleurs controversée. C'est un problème très vaste qui nécessite encore beaucoup de mesures par des techniques qui ne sont pas forcément les seules décrites aujourd'hui".

M. MOLINAR I souligne, comme suit, l'intérêt d'une utilisation plus poussée des observations que l'on pourrait faire sur le littoral français.

"Les hydrologues et les océanographes ont la chance d'avoir sur le littoral francais des estuaires et des exutoires de cours d'eau très divers vous avez tous remarqué, comme moi, que les panaches de rejet de ces exutoires sont extrêmement visibles à proximité des côtes.

"A-t-on tiré parti des observations visuelles ou photographiques que l'on pourrait entreprendre en observant la forme de ces rejets à intervalles réguliers ? Il s'agit là de mesures qui sont peu coûteuses et dont on aurait tort de se priver, parce que les conditions météorologiques varient constamment, facilitant l'échantillonnage.

"il y a là un domaine à fouiller. On commence à l'étudier d'une façon assez poussée, par exemple par des explorations systématiques au rayon nement infra-rouge. On pourrait très bien, dans beaucoup de cas, se contenter de simples photographies car la plupart des rejets pollués, y compris les rejets de rivières turbides en période de crue, sont suffisamment marqués pour qu'on en tire par ce moyen un certain nombre d'informations".

Peut être, suggère $M$. Le Président, les observations envisagées par M. MOLINAR I devraient-elles être faites, à intervalles réguliers et sur un certain nombre de points caractéristiques, à la manière de celles poursuivies depuis longtemps par les Hydrologues sur les réseaux de base des stations de jaugeage des cours d'eau.

M. Le Président clôt la discussion en remerciant M. QUETIN et toutes les personnes qui, par leur intervention, ont animé celle-ci.

Il donne ensuite la parole à M. MA R GRIT A pour l'exposé de la Communication qu'il a préparée en collaboration avec Messieurs GUIZERIX et MOLINARI. 


\section{Abstract}

\section{Use of tracers for designing and locating sea outfalls}

A thorough knowledge of hydrodynamics in the outfall area is necessary in order to estimate within a few hours the extension of polluted water discharged into the sea. Several aspects must be distinguished : tur. bulent diffusion, convection, differential convection due to variables of diverse origin : topography - wind.

The sea measurements for assessing these factors are taken with the help of "tracers" which can be radioactive, chemical (rhodamine B), or constituted drag floats.

Taking into account the problematical character of the phenomena being studied, the measurements must be numerous and simultaneous, implying a well adapted technology.

Floats are a simple and economical means of measuring trajectories at shallow depth. They must, however, be precision designed and must not offer any resistance to the wind. Unfortunately, they are not easily seen and a small aeroplane is useful for locating them in the sea. The long life of floats is an advantage for long-term measurements.

Rhodamine $B$ is used for measuring turbulent diffusion in the sea, with in particular, the taking of aerial photographs to follow the progress of the coloured stain.
Rhodamine B's optical characteristics show that it would not be easily seen at a depth of a few metres.

It is demonstrated (equations 1 and 2) that simulation of a steady outfall would be difficult, as it is unrealistic to try to obtain steady flow conditions. On the other hand, instantaneous point injections (Dirac Function) are a rapid and efficient solution.

It must be emphasized that propeller-driven boats should not be allowed to pass through the stain, as these would create much greater turbulence than that which has to be measured.

Rhodamine samples are necessary in order to assess vertical diffusion. With aerial photographs, it is possible to assess transverse eddy diffusivity, using formula 4 . However, taking account of the fact that the photograph records the rhodamine down to a certain depth, but with a weighting due to the absorption of light rays, a correction factor must be taken into consideration (equations 5 and 6). Equation 6 results from an adjustement by the method of least squares. It is not universal and can vary according to the optical properties of the seawater. 